\title{
Educação Permanente em Saúde: relato de experiência do desenvolvimento de questionário avaliativo online
}

\author{
Permanent Health Education: experience report of the development of online assessment \\ questionnaire
}
Educación Permanente en Salud: informe de la experiencia del desarrollo del cuestionario de evaluación en línea

Daniele Knopp Ribeiro ${ }^{1 *}$, Denise Barbosa de Castro Friedrich ${ }^{1}$, Edna Aparecida Barbosa de Castro', Fábio da Costa Carbogim', Zuleyce Maria Lessa Pacheco', Maria Tereza Ramos Bahia $^{1}$, Anna Karla Nascimento Lima' ${ }^{1}$, Louise Cândido Souza'1, Lucas Roque Matos ${ }^{1}$, Edith Monteiro de Oliveira ${ }^{1}$, William Ávila de Oliveira Silva ${ }^{1}$, Larissa Cristina Magalhães ${ }^{1}$, João André Tavares Álvares da Silva², Elaine Cristina Dias Franco ${ }^{3}$.

\section{RESUMO}

Objetivo: Socializar o desenvolvimento de um questionário online, produzido em contribuição de docentes e discentes de Enfermagem. Relato de experiência: Trata-se de um relato de experiência da elaboração do questionário online que foi utilizado na pesquisa "Educação Permanente como dispositivo de gestão do Sistema Único de Saúde", construído em três seções: identificação dos participantes, compreensão e aplicação da temática ao objeto do estudo e análise do formulário, em contribuição de docentes e discentes de Enfermagem. Sustentada no referencial teórico de Educação Permanente em Saúde, da Hermenêutica Dialética e das Tecnologias da Informação e Comunicação - TICs. E aplicado às referências técnicas de diversas áreas da Secretaria de Estado de Saúde de Minas Gerais e suas unidades regionais - gerências e superintendências regionais de saúde, implicados na gestão de projetos e programas desenvolvidos junto à Atenção Primária à Saúde. Considerações finais: O questionário online enquanto uma possível ferramenta para avaliação da Educação Permanente em Saúde, minimiza as limitações geográficas e consente à evolução das tecnologias de comunicação e informação.

Palavras-chave: Educação Continuada, Inquéritos e Questionários, Coleta de Dados.

\begin{abstract}
Objective: Socialize the development of an online questionnaire, made in contribution by Nursery school students and professors. Experience report: Consists of the elaboration of the online questionnaire which it was used in the research "Educação Permanente como dispositivo de gestão do Sistema Único de Saúde", built in three sections: Participants identification, comprehension and applying the theme of the object of study and the form analysis, in contribution with nursery students and professors. Based on the theoretical referential of Permanent Education in Healthcare, Hermeneutics Dialects and from Information and Communication Technologies - TICs. And applied to technical references from various areas of the Health Secretariat of the State of Minas Gerais and its regional units - regional healthcare managements and superintendence, implied in the management of projects and programs developed together with Primary Health Care. Final
\end{abstract}

1 Universidade Federal de Juiz de Fora (UFJF), Juiz de Fora-MG. *E-mail: daniele.knopp@gmail.com.

2 Universidade Federal de Minas Gerais (UFMG), Belo Horizonte-MG.

${ }^{3}$ Universidade Federal de São João Del-Rei, (UFSJ), São João Del-Rei-MG.

Financiamento: Fundação de Amparo à Pesquisa do Estado de Minas Gerais (FAPEMIG). 04208-17 
considerations: The online questionnaire as a possible tool to evaluate the Permanent Health Care Education, minimize the geographical limitations and comply with the evolution of information and communication technologies.

Keywords: Continuing Education, Surveys and Questionnaires, Data Collection.

\section{RESUMEN}

Objetivo: Socializar el desarrollo de un cuestionario en línea, producido por profesores y estudiantes de enfermería. Informe de experiencia: Este es el informe de la elaboración del cuestionario en línea que se utilizó en la investigación "La educación permanente como dispositivo de gestión del Sistema Único de Salud", integrado en tres secciones: identificación de los participantes, comprensión y aplicación del tema al tema del estudio. estudio y análisis del formulario, aportado por profesores y estudiantes de enfermería, apoyado por el marco teórico de Educación permanente en salud, hermenéutica dialéctica y tecnologías de información y comunicación - TIC, y aplicado a las referencias técnicas de diversas áreas de la Secretaría de Estado. de Minas Gerais y sus unidades regionales: gerentes regionales de salud y superintendencias, involucradas en la gestión de proyectos y programas desarrollados con Atención Primaria de Salud. Consideraciones finales: El cuestionario en línea como una posible herramienta para la evaluación de la Educación Permanente en Salud, minimiza limitaciones geográficas y permite la evolución de las tecnologías de la información y la comunicación.

Palabras clave: Educación Continua, Encuestas y Cuestionarios, Recolección de Datos.

\section{INTRODUÇÃO}

O Ministério da Saúde instituiu a Política Nacional de Educação Permanente em Saúde (PNEPS), visando fortalecer o Sistema Único de Saúde (SUS) através da reflexão coletiva sobre o trabalho e oferta de instrumentos educativos para profissionais. Foi estabelecido como pressuposto da Educação Permanente em Saúde (EPS) a aprendizagem no trabalho, pelo trabalho e para o trabalho, a partir dos problemas enfrentados na realidade dos serviços (MINISTÉRIO DA SAÚDE, 2007; FRANÇA T, et al., 2017).

A aprendizagem significativa pautada na EPS, integra o aprender e o ensinar na prática diária dos profissionais de saúde, o que repercute na prática de atenção em saúde. À medida que deixa de ser uma prática meramente pedagógica e incorpora um posicionamento ético-político-metodológico, é possível alcançar mudanças das ações do trabalho em saúde, considerando este como dispositivo de aprendizagem, por meio da reflexão coletiva (ROSSETTI LT, et al.,2019).

A reflexão do trabalho em saúde produzida pela EPS pode potencializar à assistência de qualidade ao favorecer o repensar da verticalização do modelo tradicional de assistência, de maneira a fortalecer os profissionais para o cotidiano do trabalho centralizado nos usuários, na escuta de seus contextos e discursos, em prol do crescimento da resolutividade nos serviços de saúde (SILVA KR, et al., 2017; CAMPOS KFC, et al., 2019) .

Logo, a articulação da EPS com a gestão pode potencializar processos de reorganização do sistema de saúde, à medida que atua como um dispositivo de aproximação entre o cotidiano dos trabalhadores e as necessidades da população (LEMOS CLS, 2016; MACIEL JAC, et al., 2019).

Na mediação entre EPS, serviços e gestão, a avaliação torna-se dispositivo de qualidade, através de indicadores que fornecerão parâmetros rumo a eficiência do processo. $\mathrm{O}$ ato de dar voz ao profissional de saúde sobre sua percepção acerca da EPS, reforça a política de gestão compartilhada e o compromisso com investimento em políticas de qualificação profissional (MACIEL JAC, et al., 2019).

Considerando que as Tecnologias de Informação e Comunicação (TICs) modificam a sociedade, a forma como nos comunicamos e interagimos, é importante destacar o necessário envolvimento das TICs em pesquisas na área da enfermagem, saúde e de políticas públicas de organização do SUS, como a Educação 
Permanente em Saúde, tendo em vista a diversidade e extensão territorial do Brasil (PEREIRA DM e SILVA GS, 2012; MINISTÉRIO DA SAÚDE, 2017).

Logo, o relato desta experiência tem por objetivo socializar o desenvolvimento de um questionário online, produzido em contribuição de docentes e discentes de Enfermagem.

\section{RELATO DE EXPERIÊNCIA}

Trata-se do relato da elaboração do questionário online que foi utilizado na pesquisa "Educação Permanente como dispositivo de gestão do Sistema Único de Saúde", contemplada pela Chamada 07/2017 do Programa de Pesquisa para o Sistema Único de Saúde (PPSUS) - Ministério da Saúde via Fundação de Amparo a Pesquisa de Minas Gerais (FAPEMIG), desenvolvida entre outubro de 2017 a junho 2019.

O questionário online foi construído em três seções: identificação dos participantes, compreensão e aplicação da temática ao objeto do estudo e análise do formulário.

A primeira seção destinou-se à caracterização do perfil dos participantes, incluindo a formação e qualificação profissional. Na segunda seção, foram elaboradas oito perguntas a respeito da temática Educação Permanente em Saúde como um dispositivo de gestão do SUS. Optou-se pelo formato de questionário semiestruturado, pois de acordo com Minayo MCS (2014), nele o participante tem a possibilidade de discorrer livremente sobre o tema proposto, sem condições prefixadas pelo pesquisador. A terceira e última seção, destinou-se à captação da avaliação do participante a respeito do questionário online, como uma ferramenta na coleta de dados. Nesta etapa, o profissional relatou como foi para ele, participar da pesquisa oportunizando-lhe sugerir alterações para o aperfeiçoamento do instrumento em futuras investigações.

Após a construção do questionário online, houve uma primeira validação. Esta etapa foi realizada por docentes (mestre e doutores especialistas na área de educação em saúde) e discentes participantes do grupo de pesquisa em julho de 2018.

Os docentes e discentes apresentaram suas percepções e sugeriram aperfeiçoamentos, que foram analisados pela equipe de pesquisa e implementados de acordo com sua pertinência ao objeto e método do trabalho.

No quadro 1 abaixo está o modelo do questionário em sua primeira versão online.

Quadro 1 - Primeira Versão do Questionário Online.

\begin{tabular}{|l|}
\hline \multicolumn{1}{|c|}{ Pesquisa sobre a Educação Permanente como Dispositivo de Gestão do SUS } \\
\hline \multicolumn{1}{|c|}{ Primeira Seção } \\
\hline I. Identificação dos participantes \\
Nome completo* \\
E-mail* \\
Sexo: Masculino ou Feminino * \\
Idade* \\
SRS/GRS* \\
II. Formação acadêmica \\
Área de formação da graduação: * \\
Ano de conclusão: * \\
Natureza da Instituição em que concluiu a graduação: Pública ou Privada* \\
Possui Pós-Graduação? Sim ou não** \\
Se sim, especifique: Lato sensu ou Stricto sensu \\
Caso se aplique, cite a especialidade e a data de formação. \\
Natureza da Instituição de conclusão da Pós-Graduação: Pública ou Privada \\
\hline
\end{tabular}

REAS/EJCH | Vol.11(18) | e1644 | DOI: https://doi.org/10.25248/reas.e1644.2019 Página 3 de 8 


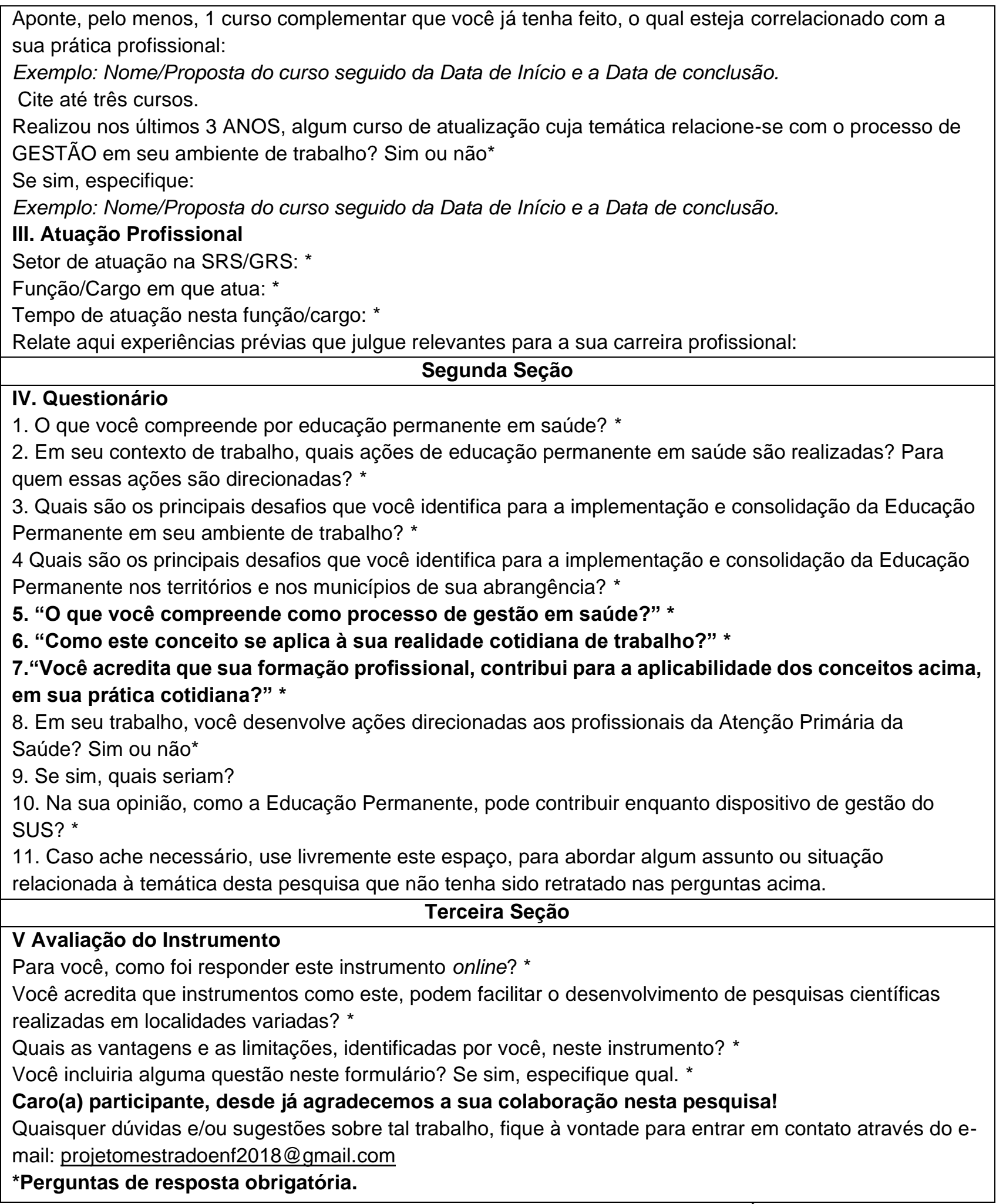

Fonte: Projeto Individual de Pesquisa (APQ) 04203/17 Programa Pesquisa para o Sistema Único de Saúde (PPSUS)/ Fundação de Amparo à Pesquisa do Estado de Minas Gerais (FAPEMIG), 2017.

Legenda: * = Questões obrigatórias de serem respondidas; SRS/GRS = Superintendências Regionais de Saúde/Gerências Regionais de Saúde; SUS = Sistema Único de Saúde.

Em continuidade ao processo de validação, o questionário online foi apresentado no mês de outubro de 2018 a mais três especialistas no tema. Todos atuantes na Secretaria de Estado de Saúde de Minas Gerais 
(SES-MG). Dois responderam ao questionário online e o terceiro discutiu as questões e formatação com a equipe de pesquisa.

Com resultado deste processo algumas questões da segunda seção do questionário online, subdivisão IV, foram alteradas. São elas:

A questão cinco: "O que você compreende como processo de gestão em saúde?" Foi alterada para "O que você compreende por gestão em saúde?", pois foi observado que a presença da palavra "processo" modificou o objetivo da questão.

A questão seis: "Como este conceito se aplica à sua realidade cotidiana de trabalho?" Foi alterada para: "Como a gestão em saúde se aplica em sua realidade cotidiana de trabalho?", pois as respostas não abordaram de forma completa o questionamento da pergunta.

A questão sete: "Você acredita que sua formação profissional, contribui para a aplicabilidade dos conceitos acima, em sua prática cotidiana?" Foi alterada para: "Como sua formação profissional, contribui para a aplicabilidade dos conceitos acima, em sua prática cotidiana?", por entender que o texto anterior gerava dúvidas para os respondentes.

Por fim, todo o material foi encaminhado para o último especialista da SES-MG, que teve dúvidas quanto à questão oito: "Em seu trabalho, você desenvolve ações direcionadas aos profissionais da Atenção Primária da Saúde (APS)?". Questionou a equipe de pesquisa se outros profissionais de outro nível de atenção, não participariam. A equipe informou que os participantes seriam aqueles que atuavam com a APS. A questão não foi alterada. Já as alterações relativas à formatação do documento foram incorporadas.

A versão final do questionário online, foi produzido por meio da Plataforma Google, utilizando suas ferramentas online Google Forms e Google Docs. CASTELANE OO, et al. (2017), ressalta que o Google Forms possibilita a customização de formulários pelos autores de acordo com seus objetivos, além disso, os dados coletados são automaticamente organizados em planilhas e gráficos, permitindo que todo o processo seja online, com acesso controlado mediante convite via e-mail e armazenamento na nuvem do Google.

O quadro II mostra a versão final do questionário online que foi enviado para os 151 participantes da pesquisa. Sendo estes, referências técnicas de diversas áreas da SES-MG e suas unidades regionais gerências e superintendências regionais de saúde, implicados na gestão de projetos e programas desenvolvidos junto à Atenção Primária à Saúde.

Quadro 2 - Versão Final do Questionário Online.

\begin{tabular}{|l|}
\hline \multicolumn{1}{|c|}{ Pesquisa sobre a Educação Permanente como Dispositivo de Gestão do SUS } \\
\hline \multicolumn{1}{|c|}{ Primeira Seção } \\
\hline I. Identificação dos participantes \\
Nome completo* \\
E-mail* $^{*}$ Sexo: Masculino ou Feminino * \\
Idade* \\
SRS/GRS* \\
II. Formação acadêmica \\
Área de formação da graduação: * \\
Ano de conclusão: * \\
Natureza da Instituição em que concluiu a graduação: Pública ou Privada* \\
Possui Pós-Graduação? Sim ou não* \\
Se sim, especifique: Lato sensu ou Stricto sensu \\
Caso se aplique, cite a especialidade e a data de formação. \\
Natureza da Instituição de conclusão da Pós-Graduação: Pública ou Privada \\
Aponte, pelo menos, 1 curso complementar que você já tenha feito, o qual esteja correlacionado com a \\
sua prática profissional: \\
\hline
\end{tabular}

REAS/EJCH | Vol.11(18) | e1644 | DOI: https://doi.org/10.25248/reas.e1644.2019 Página 5 de 8 


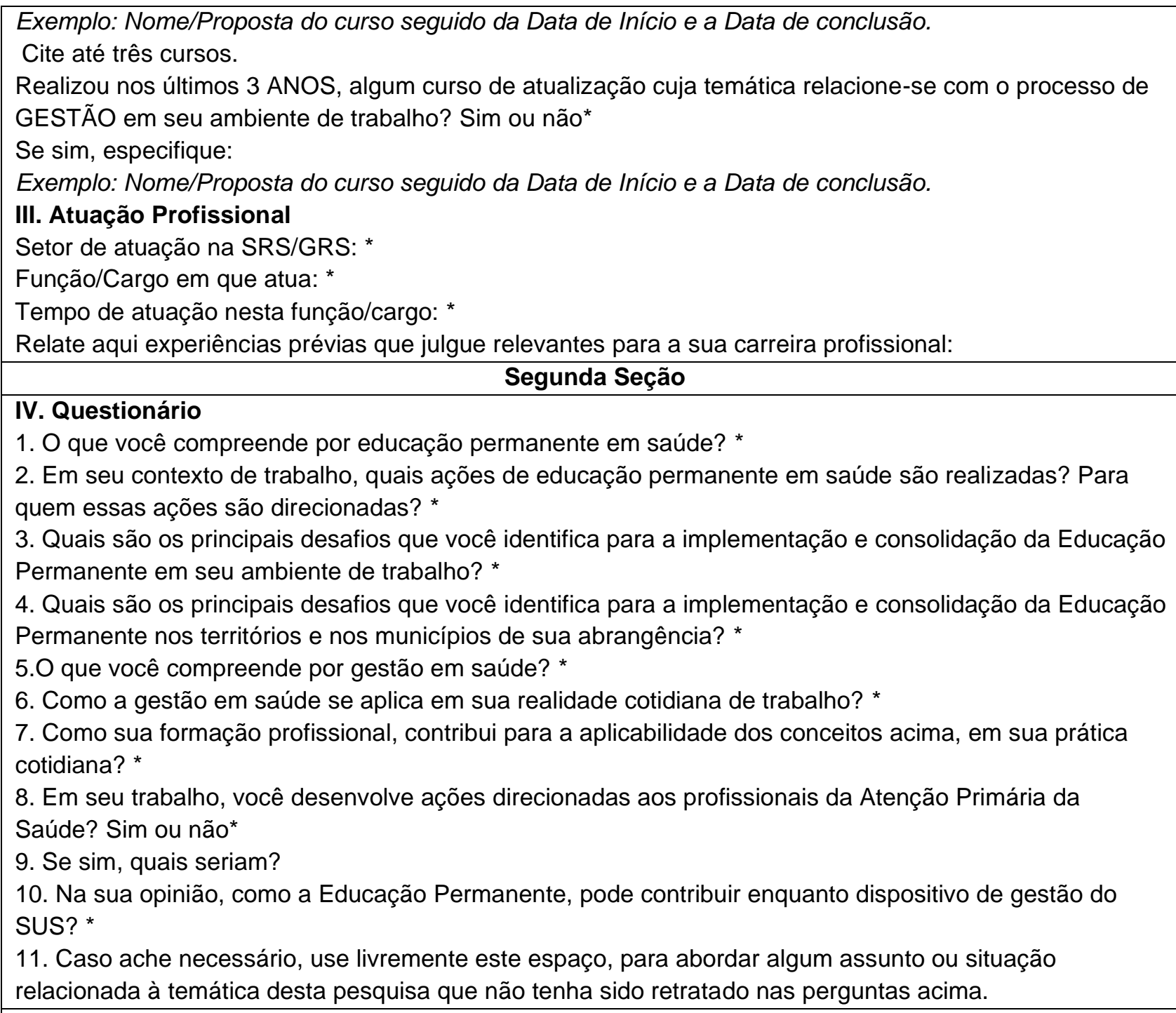

\section{Terceira Seção}

\section{Avaliação do Instrumento}

Para você, como foi responder este instrumento online? *

Você acredita que instrumentos como este, podem facilitar o desenvolvimento de pesquisas científicas realizadas em localidades variadas? *

Quais as vantagens e as limitações, identificadas por você, neste instrumento? *

Você incluiria alguma questão neste formulário? Se sim, especifique qual. *

Caro(a) participante, desde já agradecemos a sua colaboração nesta pesquisa!

Quaisquer dúvidas e/ou sugestões sobre tal trabalho, fique à vontade para entrar em contato através do email: projetomestradoenf2018@gmail.com

${ }^{*}$ Perguntas de resposta obrigatória

Fonte: Projeto Individual de Pesquisa (APQ) 04203/17 Programa Pesquisa para o Sistema Único de Saúde (PPSUS)/ Fundação de Amparo à Pesquisa do Estado de Minas Gerais (FAPEMIG) (2017).

Legenda: * = Questões obrigatórias de serem respondidas; SRS/GRS = Superintendências Regionais de Saúde/Gerências Regionais de Saúde; SUS = Sistema Único de Saúde.

\section{DISCUSSÃO}

Buscou-se, através de revisão de literatura inicial a aproximação de conceitos e especificidades da EPS como política pública e possível dispositivo de gestão. Dessa forma, foi extraída da literatura informações que possibilitasse a compreensão do conceito e os vinculasse a elaboração de formulário online. 
Uma parceria acadêmica ocorreu entre mestrandos, bolsistas de desenvolvimento tecnológico e incentivo à inovação, bem como de iniciação científica da Faculdade de Enfermagem da Universidade Federal de Juiz de Fora.

O trabalho da equipe esteve atrelado à busca por um questionário robusto e sustentado no referencial teórico de educação permanente em saúde (MINISTÉRIO DA SAÚDE, 2007), da hermenêutica dialética (MINAYO MCS, 2014) e das Tecnologias da Informação e Comunicação - TICs (FALEIROS F, et al., 2016).

Através da problematização da hermenêutica dialética, o grupo discutia os dados extraídos da revisão da literatura realizada sobre educação permanente em saúde, além das TICs. Os encontros propiciaram um rico processo de aprendizagem significativa, pois as discussões se baseiam em problemas como divergências entre autores e experiências pessoais dos participantes.

Com o acesso crescente à internet em todo o mundo, as pesquisas com o uso do ambiente virtual mostramse como uma tendência atual para a coleta de dados. Assim, a utilização da internet, como recurso auxiliar de troca e disseminação de informações, possibilita a melhoria e a agilidade do processo de investigação. Além de permitir ao pesquisador ter acesso aos participantes de diferentes localidades, em um curto espaço de tempo, e a um baixo custo operacional (FALEIROS F, et al., 2016).

Para Medeiros RKS, et al. (2015), a importância da qualidade dos instrumentos que procuram verificar e avaliar um fenômeno é fundamental para a legitimidade e credibilidade dos resultados de uma pesquisa. Sob este pressuposto, o questionário online foi construído e validado.

A construção do questionário online se sustentou na necessidade de um dispositivo de avaliação da educação permanente em saúde, atrelado à evolução das tecnologias de comunicação e informação.

A interatividade e a dinâmica, inerentes às tecnologias de comunicação e informação, são importantes para a realização de pesquisas no âmbito local, regional e nacional, independente das limitações geográficas. Através da apresentação do questionário online, enquanto uma possível ferramenta para avaliação, ressaltase a necessidade da articulação entre o ensino, pesquisa, gestão e serviços para o aprimoramento conjunto da educação permanente em saúde.

\section{CONSIDERAÇÕES FINAIS}

A PNEPS é uma significativa política pública de fortalecimento dos trabalhadores de saúde para o cotidiano de seu trabalho e apoio a qualificação da assistência prestada à sociedade. Dar voz aos profissionais de saúde é possibilitar a reflexão sobre o trabalho, da gestão de projeto e programas ao cuidado nos serviços de saúde. Considerando o lugar de destaque que a internet tem ocupado na produção e comunicação de informações, a utilização de questionário online nas investigações científicas é uma possibilidade frente a limitações geográficas, tempo e recursos financeiros.

\section{REFERÊNCIAS}

1. CAMPOS KFC, et al. Educação Permanente em Saúde e modelo assistencial: correlações no cotidiano de serviço na Atenção Primária à Saúde. APS em Revista, 2019; 1(2): 132-140.

2. CASTELANE OO, et al. Contribuição da plataforma Google nas práticas pedagógicas em torno da produção textual. Revista Intersaberes, 2012; 12: 402-414.

3. FALEIROS $\mathrm{F}$, et al. Uso de questionário online e divulgação virtual como estratégia de coleta de dados em estudos científico. Texto e Contexto Enfermagem, 2016; 25: 1-6.

4. FRANÇA T, et al. Política de Educação Permanente em Saúde no Brasil: a contribuição das Comissões Permanentes de Integração Ensino-Serviço. Ciência e Saúde Coletiva, 2017; 22: 1817-1828.

5. LEMOS CLS. Educação Permanente em Saúde no Brasil: educação ou gerenciamento permanente? Ciência e Saúde Coletiva, 2016; 21(3): 913-922.

6. MACIEL JAC, et al. Discurso do sujeito coletivo das concepções sobre educação permanente em saúde de gestores e cirurgiões-dentistas da atenção primária à saúde. Revista Pesquisa Qualitativa, 2019; 7:117-134. 
7. MEDEIROS RKS, et al. Modelo de validação de conteúdo de Pasquali nas pesquisas em Enfermagem. Revista de Enfermagem Referência, 2015; 4: 127-135.

8. MINAYO MCS. O Desafio do Conhecimento: Pesquisa Qualitativa em Saúde.14ª ed. São Paulo: Hucitec, $2014 ; 408$ p.

9. MINISTÉRIO DA SAÚDE. Portaria ํㅜ 1.996, de 20 de agosto de 2007. Dispõe sobre as diretrizes para a implementação da Política Nacional de Educação Permanente em Saúde.

10. MINISTÉRIO DA SAÚDE. Portaria de consolidação no 2, de 28 de setembro de 2017. Consolida as normas sobre as políticas nacionais de saúde do Sistema Único de Saúde.

11. PEREIRA DM, SILVA GS. As Tecnologias de Informação e Comunicação (TICs) como aliadas para o desenvolvimento. Cadernos de Ciências Sociais Aplicadas, 2012; 7(8): 2358-1212.

12. ROSSETTI LT, et al. Educação permanente e gestão em saúde: a concepção de enfermeiros. Revista Online de Pesquisa: Cuidado é Fundamental, 2019; 11: 129-134.

13. SILVA KL, et al. A construção da educação permanente no processo de trabalho em saúde no estado de Minas Gerais, Brasil. Escola Anna Nery Revista de Enfermagem, 2017; 21(4): 1-8. 